\title{
MyD88 is an essential component of retinoic acid-induced differentiation in human pluripotent embryonal carcinoma cells
}

\author{
Gomaa Sulaiman ${ }^{1,2,11}$, Aoife Cooke ${ }^{1,2,11}$, Brendan Ffrench ${ }^{1,2}$, Claudia Gasch ${ }^{1,2}$, Olayemi Azeez Abdullai ${ }^{1,2}$, Kevin O'Connor $^{1,2}$, \\ Salah Elbaruni ${ }^{1,2}$, Gordon Blackshields ${ }^{1}$, Cathy Spillane ${ }^{1,2}$, Helen Keegan ${ }^{1,2}$, Victoria McEneaney ${ }^{1,2}$, Ronan Knittel ${ }^{1,2}$, \\ Annamarie Rogers ${ }^{3}$, Ian B Jeffery ${ }^{4}$, Brendan Doyle ${ }^{1,2}$, Mark Bates ${ }^{1}$, Charles d'Adhemar ${ }^{1,2}$, Mathia YC Lee ${ }^{5}$, Eric L Campbell ${ }^{6}$, \\ Paul N Moynagh ${ }^{7,6}$, Desmond G Higgins ${ }^{8}$, Sharon O'Toole ${ }^{1,9}$, Luke O'Neill ${ }^{10}$, John J O'Leary ${ }^{1,2}$ and Michael F Gallagher ${ }^{\star, 1,2}$
}

We have previously reported that myeloid differentiation primary response gene 88 (MyD88) is downregulated during all-trans retinoic acid (RA)-induced differentiation of pluripotent NTera2 human embryonal carcinoma cells (hECCs), whereas its maintained expression is associated with RA differentiation resistance in nullipotent 2102Ep hECCs. MyD88 is the main adapter for toll-like receptor (TLR) signalling, where it determines the secretion of chemokines and cytokines in response to pathogens. In this study, we report that loss of MyD88 is essential for RA-facilitated differentiation of hECCs. Functional analysis using a specific MyD88 peptide inhibitor (Peplnh) demonstrated that high MyD88 expression in the self-renewal state inhibits the expression of a specific set of HOX genes. In NTera2 cells, MyD88 is downregulated during RA-induced differentiation, a mechanism that could be broadly replicated by MyD88 Peplnh treatment of 2102Ep cells. Notably, MyD88 inhibition transitioned 2102Ep cells into a stable, selfrenewing state that appears to be primed for differentiation upon addition of RA. At a molecular level, MyD88 inhibition combined with RA treatment upregulated HOX, RA signalling and TLR signalling genes. These events permit differentiation through a standard downregulation of Oct4-Sox2-Nanog mechanism. In line with its role in regulating secretion of specific proteins, conditioned media experiments demonstrated that differentiated (MyD88 low) NTera2 cell media was sufficient to differentiate NTera2 cells. Protein array analysis indicated that this was owing to secretion of factors known to regulate angiogenesis, neurogenesis and all three branches of TGF- $\beta$ Superfamily signalling. Collectively, these data offer new insights into RA controlled differentiation of pluripotent cells, with notable parallels to the ground state model of embryonic stem cell self-renewal. These data may provide insights to facilitate improved differentiation protocols for regenerative medicine and differentiation-therapies in cancer treatment.

Cell Death and Differentiation (2017) 24, 1975-1986; doi:10.1038/cdd.2017.124; published online 8 September 2017

Pluripotent stem cells have great regenerative medicine potential owing to their ability to differentiate into tissues representative of all three germ layers. ${ }^{1-3}$ Pluripotency remains imprecisely characterised, particularly the events upstream of the key regulatory trio Oct4, Sox2 and Nanog. It has been reported that pluripotent embryonic stem cells (ESCs) transition from a naive 'ground state' through primed states towards commitment to various specific lineages. ${ }^{4-6}$ This process appears to be tightly regulated by the presence of specific growth factors within the niche, particularly those regulating the three branches of TGF- $\beta$ Superfamily (BMP, TGF- $\beta$ and activin) signalling. ${ }^{7,5}$ Harnessing the power of pluripotent stem cells requires an improved understanding of this regulatory mechanism.
As they are more stable in culture than human ESCs (hESCs) or induced pluripotent stem cells (iPSCs), human embryonal carcinoma cells (hECCs) are a useful tool for the elucidation of pluripotent mechanisms. ${ }^{8-11}$ We have previously reported that myeloid differentiation response gene 88 (MyD88) expression is downregulated during retinoic acid (RA)-induced differentiation of pluripotent NTera2 hECCS but maintained in RA-treated nullipotent 2102Ep hECCs. ${ }^{12}$ The RA signalling pathway initially involves recognition and translocation of retinoids by cell surface receptor STRA6 (Stimulated by RA6), followed by translocation through the cell via Cellular Retinoid and Cellular Retinoic Acid Binding Proteins (CRBPs \& CRABPs). Subsequently, RA binds to

\footnotetext{
${ }^{1}$ Department of Histopathology, Trinity College Dublin, Central Pathology Laboratory, St James's Hospital, Dublin 8, Ireland; ${ }^{2}$ Pathology Research Laboratory, Coombe Women and Infant's University Hospital, Dublin 8, Ireland; ${ }^{3}$ Department of Applied Sciences, Dundalk Institute of Technology, Dublin Road, Dundalk, Co. Louth, Ireland; ${ }^{4}$ APC Institute and Department of Microbiology, University College Cork, Cork, Ireland; ${ }^{5}$ Graduate School for Integrative Sciences and Engineering, National University of Singapore, Singapore, Singapore; ${ }^{6}$ Centre for Experimental Medicine, Queen's University Belfast BT9 ZBL, Northern Ireland, UK; ${ }^{7}$ Institute of Immunology, Department of Biology, Maynooth University, Maynooth, Kildare, Ireland; ${ }^{8}$ School of Medicine and Conway Institute, University College Dublin, Belfield, Dublin 4, Ireland; ${ }^{9}$ Department of Obstetrics and Gynaecology, Trinity College Dublin, Trinity Centre for Health Sciences, St James's Hospital, Dublin 8, Ireland and ${ }^{10}$ School of Biochemistry and Immunology, Trinity Biomedical Science Institute, Trinity College Dublin, Dublin 2, Ireland

${ }^{*}$ Corresponding author: MF Gallagher, Department of Histopathology, Trinity College Dublin, Central Pathology Laboratory, St James's Hospital, Dublin, Dublin 8, Ireland. Tel/Fax: +353 140855 26; E-mail: gallagmi @tcd.ie

${ }^{11}$ These authors contributed equally to this work.

Received 27.1.17; revised 02.6.17; accepted 19.6.17; Edited by R De Maria; published online 08.9.17
} 

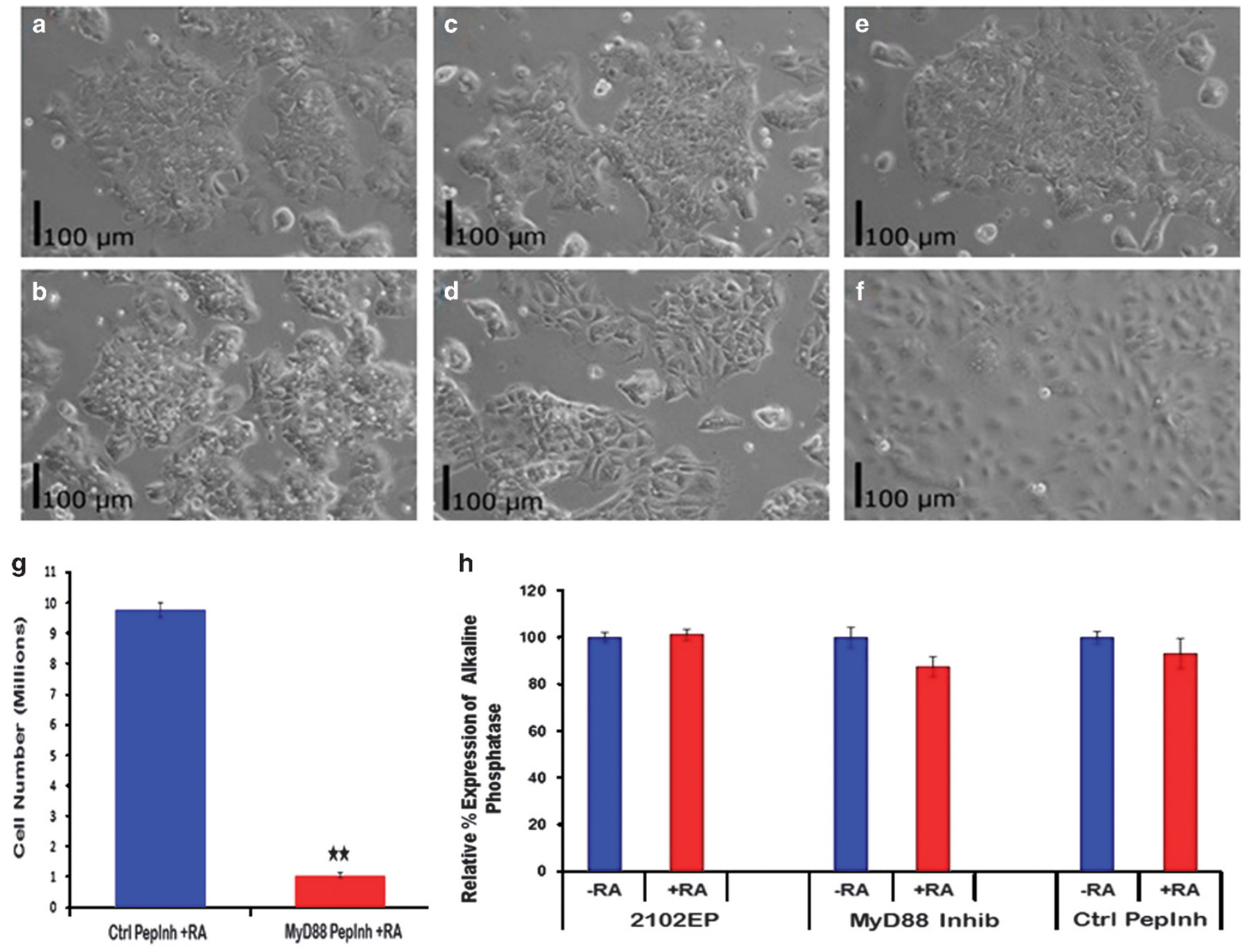

i
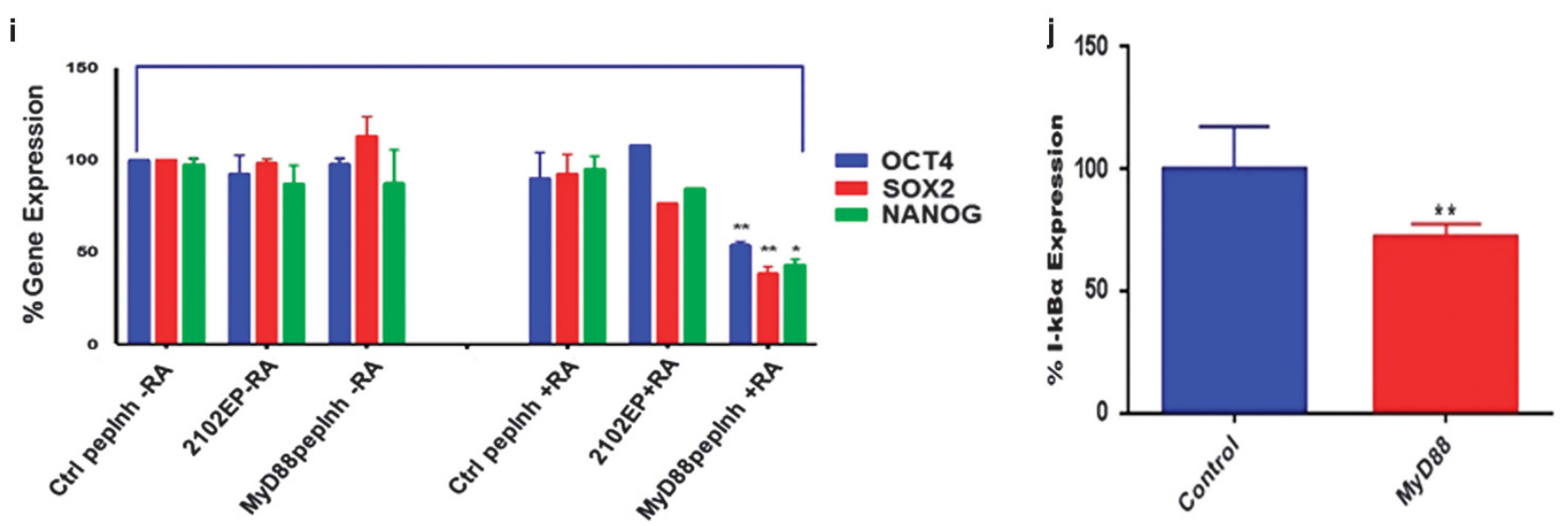

Figure 1 Loss of MyD88 facilitates RA-induced differentiation of a sub-population of 2102Ep cells. 2102Ep cells were treated with MyD88 Peplnh for $24 \mathrm{~h}$ and then with MyD88 Peplnh+RA (refreshed daily) for an additional 11 days. After these 12 days treatment, cellular morphology was found to be unaltered in control cells (a: - RA, $\mathbf{b}$ : +RA, c: control Peplnh - RA, d: control Peplnh +RA e: MyD88 Pepinh-RA. All $\times 10$ magnification), suggesting that these cells remained undifferentiated. In contrast, the majority of cells grown in MyD88 Peplnh+RA conditions demonstrated a differentiated morphology (f). This was supported by cell count data indicating a substantial, significant decrease in MyD88 Peplnh+RA-treated cells compared with controls (g) but was contradicted by similar levels of pluripotency marker AP measured by quantitative ELISA (h). These samples were harvested and their differentiation status confirmed through qPCR analysis of Oct4, Sox2 and Nanog levels (i), which indicated that the levels of these pluripotency markers had not decreased in MyD88 Peplnh+RA cells sufficiently to indicate typical hECC differentiation based on our typical data. ${ }^{45}$ Finally, $24 \mathrm{~h}$ treatment with the MyD88 Peplnh was shown to result in a statistically significant decrease in phospho- $\mid \kappa B$ expression as measured by quantitative ELISA following treatment with Interleukin- $1 \beta$, indicating a decrease in MyD88-dependent TLR signalling (j). Together, these data led to a hypothesis that MyD88 Peplnh+RA samples contain two sub-populations, one differentiated and one nullipotent, the testing of which is described in Figure $2 .{ }^{*} P$-value $<0.05$; ${ }^{*} P$-value $<0.01$ 
nuclear RA and Retinoid X Receptors (RARs \& RXRs), which facilitate regulation of targets such as HOX genes and Oct4Sox2-Nanog. ${ }^{13-16}$ MyD88 is best known for its role as the main adapter protein for toll-like receptor (TLR) signalling, a key component of innate immunity. ${ }^{17-19}$ In response to detection of specific pathogens, MyD88-dependent TLR signalling activates NF- $\kappa \mathrm{B}$. Alternatively, MyD88-independent TLR signalling results in activation of type 1 interferons, which act through interferon-stimulated genes (ISGs) and JAK-STAT signalling. ${ }^{18,19}$ Each mechanism results in secretion of

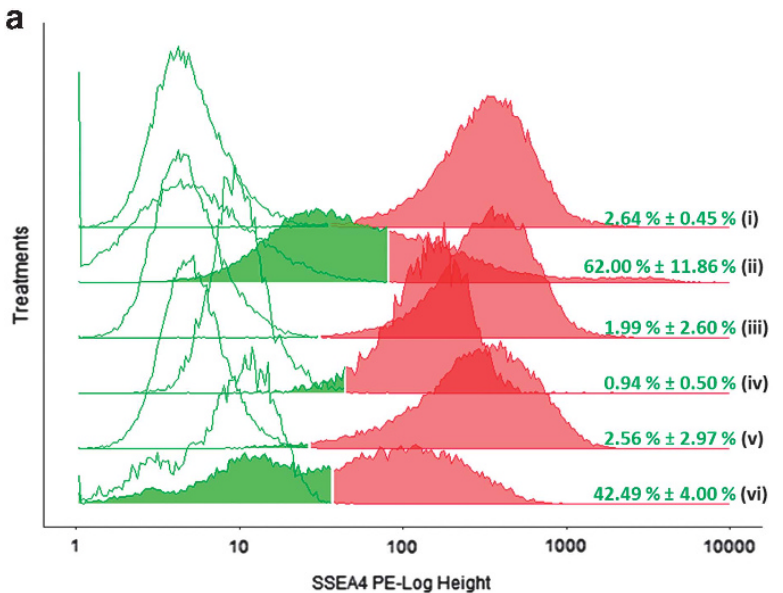

b
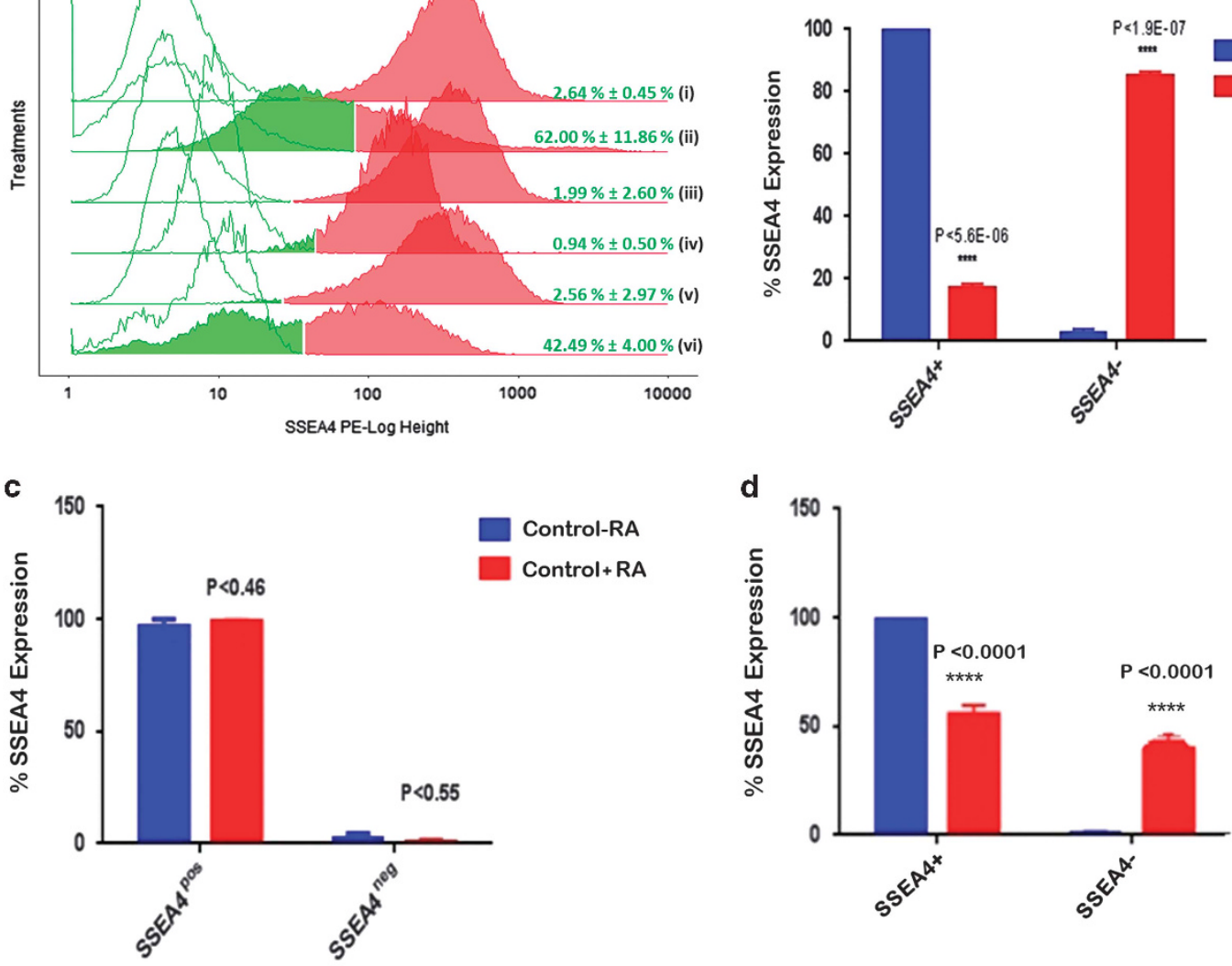

SINEG 2102EP

Si SOX2 2102EP

d

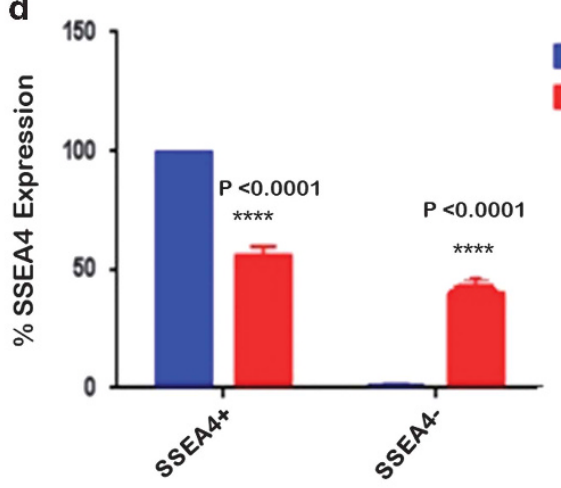

MyD88 PepInh-RA

MyD88 PepInh+RA
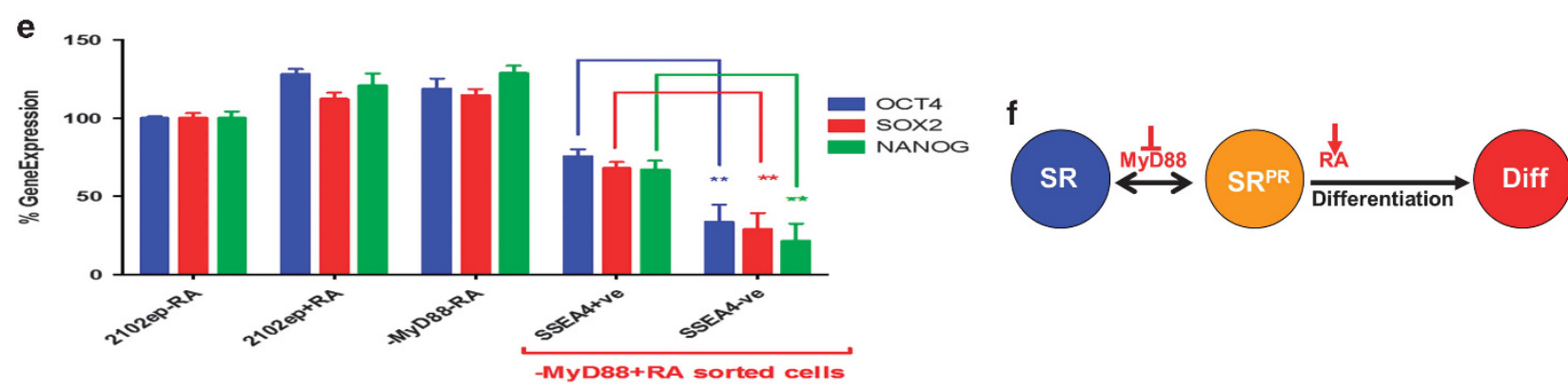

Figure 2 Inhibition of MyD88 facilitates RA-induced differentiation of 2102Ep cells. 2102Ep cells were treated with MyD88 Peplnh for $24 \mathrm{~h}$ and then with MyD88 Peplnh+RA (refreshed daily) for an additional 11 days. Cells from three independent replicates were assessed via flow cytometry (a) for differentiation status using pluripotency marker SSEA4. (a) Open lines represent negative staining controls for each sample. Filled lines represent the stained sample. Green represents the SSEA4 ${ }^{\text {Neg }}$ cells and red the SSEA4 ${ }^{\text {Pos }}$ cells, as determined by internal negative staining controls. $2102 E p$ cells were shown to be almost completely SSEA4 ${ }^{\text {Pos }}$ in $-R A$ conditions $(a-i)$ and $62.00 \pm 11.86 \%$ SSEA4 ${ }^{\mathrm{Neg}}$ when force differentiated using a siRNA knockdown of Sox2 protocol we have previously described (a-ii), ${ }^{45}$ differences that were found to be statistically significant (b). Cells grown in control Peplnh -RA (a-iii), MyD88 Peplnh -RA (a-iv) and control Peplnh +RA (a-v) conditions were found to remain almost completely SSEA4 ${ }^{\text {Pos }}$ (a-iii-v: c-d, statistical analysis). In contrast, cells grown in MyD88 Peplnh+RA conditions for 12 days were found to be 1:1 SSEA4 ${ }^{\text {Pos }}:$ SSEA4 ${ }^{\text {Neg }}$ (a-vi: $d$, statistical analysis). Although this was initially thought to suggest the presence of two sub-populations, additional experiments (Supplementary Data 4) suggest that this is owing to a small population of cells that persist through RA treatment, which can proliferate to a sizable populations over this time-scale. SSEA4 ${ }^{\text {Pos }}$ and SSEA4 ${ }^{\text {Neg }}$ populations (a-vi) were isolated via FACS and shown by qPCR to express Oct4-Sox2-Nanog at high and low levels respectively (e). Collectively, this indicates a model (f) where 2102Ep cells can be transitioned to a stable, apparently primed self-renewal $\left(\mathrm{SR}^{\mathrm{PR}}\right)$ state when MyD88 is consistently inhibited, a state that can be differentiated by $\mathrm{RA}$. ${ }^{*} P$-value $<0.05 ;{ }^{* *} P$-value $<0.01$ 
specific chemokines and cytokines, which facilitate an immune response. ${ }^{20,21}$ A relationship between MyD88 and pluripotency has not been previously described.

Here, we report that loss of MyD88 is a key component of RA-induced differentiation in hECCs. Mechanistically, MyD88 promotes self-renewal through inhibition of $\mathrm{HOX}$ and RA signalling pathway gene expression, while its loss facilitates transition to an alternative self-renewal state that is primed for RA-induced differentiation through the standard downregulation of Oct4-Sox2-Nanog mechanism. Functionally, conditioned media experiments demonstrated that factors secreted by differentiated (low MyD88) cells were sufficient to force differentiation of NTera2 cells. When characterised, these proteins were identified as known regulators of all three branches of TGF- $\beta$ Superfamily signalling, as well as angiogenesis and neurogenesis. Collectively, these data provide new insights in to the mechanisms involved in early differentiation of pluripotent hECCs.

\section{Results}

MyD88 is sufficient to maintain the self-renewal state and its loss necessary for RA differentiation. We screened early time-point data from a RA differentiation experiment and identified MyD88 as a potential upstream regulator of Oct4Sox2-Nanog (Supplementary Data 1-3). ${ }^{12}$ Hypothesising that loss of MyD88 may be necessary for RA differentiation of hECCs, nullipotent 2102Ep cells were treated with a MyD88 peptide inhibitor (Peplnh), which was refreshed daily, in combination with RA. Measurement of phosphorylated-I-KB- $\alpha$ expression in response to Interleukin-1 $\beta$ (IL-1 $\beta$ ) indicated that MyD88-dependent TLR signalling was significantly decreased by MyD88 Peplnh treatment (Figure 1j). The morphology and cellular proliferation characteristics of MyD88 Peplnh+RA-treated (12 days) cells suggested differentiation, a response that was not observed in cells treated with MyD88 Peplnh-RA or control Peplnh \pm RA (Figures 1a-f). Cells treated with MyD88 Peplnh+RA showed a substantial decrease in cellular proliferation compared with cells treated with control Peplnh+RA, which was also suggestive of differentiation (Figure 1g). Notably, MyD88 Peplnh-treated cells did not spontaneously differentiate, whereas withdrawal of the MyD88 Peplnh rendered cells resistant to RA (reversion to nullipotency). This indicates a mechanism where consistent inhibition of MyD88 transitions 2102Ep cells into a new, stable self-renewal state that is now primed for differentiation in response to RA. Flow cytometry analysis of the expression of pluripotency marker stage specific embryonic antigen 4 (SSEA4) on individual cells demonstrated the presence of approximately equal numbers of selfrenewing (SSEA4 ${ }^{\mathrm{Pos}}$ ) and differentiated (SSEA4 ${ }^{\mathrm{Neg}}$ ) cells after 12 days MyD88 Peplnh+RA treatment (Figures 2a-d). In line with this, cells harvested at the end of this experiment showed expression of pluripotency markers alkaline phosphatase (AP, Figure 1h) and Oct4, Sox2 and Nanog (Figure 1i) suggestive of a mixed population. However, when separated by FACS, SSEA4 ${ }^{\mathrm{Pos}}$ and SSEA4 ${ }^{\mathrm{Neg}}$ cells were found to express high and low levels of Oct4, Sox2 and Nanog respectively (Figure $2 e$ ), confirming the undifferentiated and differentiated states of these 2 cell types. These data indicate a model where loss of MyD88 is necessary for RA-induced differentiation of 2102Ep cells. Mechanistically, loss of MyD88 transitions the majority of 2102Ep cells to what appears to be a stable, primed self-renewal $\left(S R^{P R}\right)$ state, which facilitates differentiation upon addition of RA (Figure 2f).

At first glance, the presence of a large, undifferentiated subpopulation after 12 days treatment (Figure 2a) suggested the presence of two sub-populations within the 2102Ep model. However, it must be noted that a small population of rapidly proliferating undifferentiated cells substantially expand over time compared with slow or non-proliferating differentiated cells. With this in mind, in RA-treated NTera2 cells, we detected a small, persistent undifferentiated population, both in culture and in vivo xenografts, which could substantially expand over time (Supplementary Data 4). As such, the undifferentiated cells may represent a lack of complete efficiency in hECC differentiation protocols rather than a distinct sub-population.

As these data indicated that loss of MyD88 was required for RA-induced differentiation of 2102Ep cells, we next hypothesised that MyD88 might be sufficient for maintenance of the pluripotent NTera2 self-renewal state. Testing this, MyD88 was overexpressed in NTera2 cells using a constitutive expression plasmid, and then challenged with RA. When treated with RA, cells overexpressing MyD88 (Figures $3 a$ and b) retained substantially higher levels of pluripotency markers AP (Figure 3c), Oct4 and Nanog (Figure 3d) compared with RAtreated control cells. Collectively, these data indicate that MyD88 is sufficient to maintain the self-renewal state and its loss necessary for RA differentiation. In addition, inhibition of MyD88 can be used to generate what appears to be a stable self-renewal state that is primed for RA differentiation.

\section{A potential role for MyD88 in mesodermal differentiation} of 2102Ep cells. Once it had been shown that loss of MyD88 was necessary for RA differentiation, we assessed whether loss of MyD88 facilitated non-RA lineage differentiation mechanisms. As specific lineage differentiation protocols have not been previously optimised for hECCs, commercially available early lineage differentiation commitment kits were used. These protocols are useful indicators of initiation of differentiation, but further lineage-specific protocols are required for complete differentiation. Although the exact content of the growth factor mixes supplied with these kits is not released, the supplier confirmed for us that none contained RA. 2102Ep cells were treated with and without the MyD88 Peplnh for $24 \mathrm{~h}$ as before and then treated with specific differentiation protocols for each lineage. Differentiation was then assessed as changes in morphology, and loss of SSEA4 and Oct4-Sox2-Nanog expression. This analysis suggested that the mesoderm differentiation kit could replace RA in the MyD88 inhibited mechanism observed previously (Figures $4 \mathrm{a}-\mathrm{i}$ ). The strongest evidence for this is the obvious difference in morphology observed in cells treated with MyD88 Peplnh+mesoderm kit but not control cells (Figures $4 \mathrm{e}$ and $\mathrm{f}$ ). This is in line with previous reports linking $\mathrm{RA}$ and mesodermal differentiation of mES cells. ${ }^{22,23}$ However, although the mesoderm kit-only control had no effect on 
a
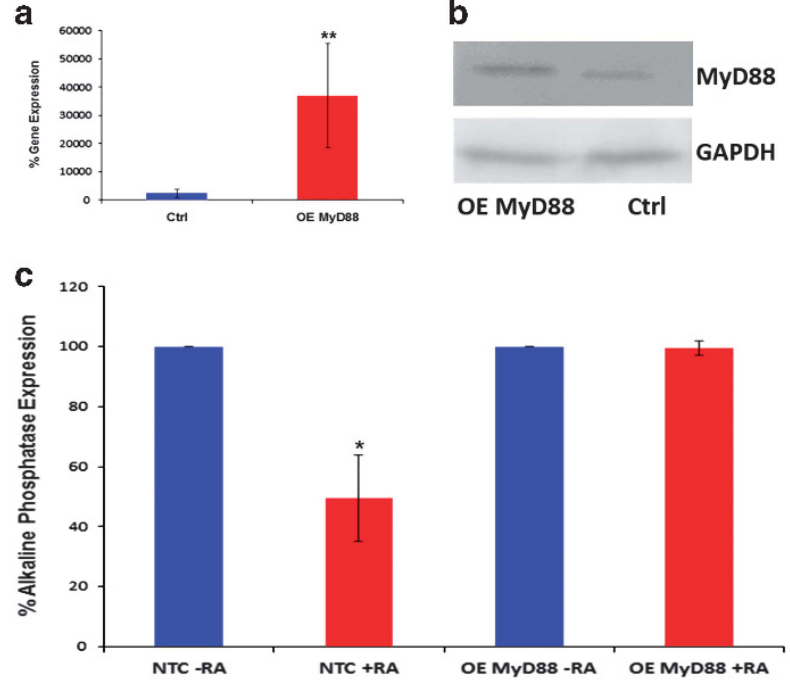

d

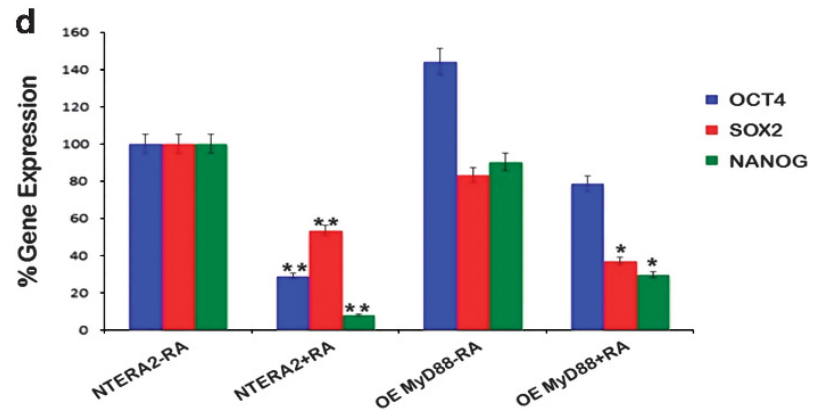

Figure 3 MyD88 overexpression inhibits RA differentiation of NTera2 cells. MyD88 was overexpressed (4 days) in NTera2 cells using a constitutive expression plasmid. Overexpression of MyD88 was confirmed by qPCR (a: $P=0.004)$ and western blot (b) compared with empty plasmid and transfection reagent only controls (Ctrl) respectively. (c) The expression of pluripotency marker Alkaline Phosphatase (AP) as measured by quantitative ELISA was significantly decreased in nontransfected control (NTC) cells treated with RA (4 days) compared with -RA-treated NTC cells. In contrast, cells overexpressing MyD88 (OEMyD88) showed similar expression of $\mathrm{AP}$ in $\pm \mathrm{RA}$ conditions. (d) $\mathrm{PPCR}$ analysis of these samples demonstrated that Oct4 $(P=0.004)$ and Nanog $(P=0.02)$ expression was not downregulated in MyD880E+RA cells in a fashion observed for NTC+RA cells (Sox2 is lower in MyD880E+RA cells, $P=0.03$ ). These data indicate that maintained expression of MyD88 is sufficient to inhibit RA-induced differentiation of NTera2 cells. ${ }^{*} P$-value $<0.05 ;{ }^{* \star} P$-value $<0.01$

morphology (Figure 4e), flow cytometry using SSEA4 revealed two populations with only a small, statistically significant difference compared with MyD88 Peplnh+kit (Figures $4 \mathrm{~g}$ and $\mathrm{h}$ ). In contrast, the ectoderm differentiation protocol resulted in the production of an SSEA4 ${ }^{\mathrm{Neg}}$ subpopulation that was too small to convincingly suggest a functional differentiation mechanism (Figures $4 \mathrm{~g}, \mathrm{I}$ and $\mathrm{m}$ ). The endoderm differentiation protocol did not result in the differentiation of any 2102Ep cells (Figures $4 \mathrm{~g}, \mathrm{j}$ and $\mathrm{k}$ ). These morphology results suggest that MyD88 regulation is not RA-specific and may have a role in mesoderm differentiation, but further studies are required to confirm this.

Differentiated NTera2 cells secrete factors sufficient for differentiation. The main known role of MyD88 is in TLR Signalling, where it determines the profile of factors secreted by the cell in response to specific stimuli. Addressing this, we next undertook a conditioned media experiment to assess whether self-renewing (high MyD88) and differentiated (low MyD88) hECCs secrete a different set of factors that influence their stem cell status. Owing to the nullipotent status of the 2102Ep cells, NTera2 cells were primarily used in this conditioned media analysis. We first established that NTera2 cells remain terminally differentiated following 7 days RA-free treatment, even when RA is withdrawn (Supplementary Data 4). Differentiated NTera2 cells could thus be grown in RA-free media, which could be conditioned with factors secreted by differentiated cells without RA contamination. Conditioned media collected for either 7 or 14 days from undifferentiated ('UndiffCon') and differentiated ('DiffCon') NTera2 cells was added to NTera2 cells daily for 7 days. Based on their subsequent expression of SSEA4, Oct4, Sox2 and Nanog, treatment of NTera2 cells with DiffCon (collected for 7 or 14 days) media was found to be sufficient to induce differentiation of NTera2 cells (Figures $5 \mathrm{a}-\mathrm{C}$ ). In contrast, UndiffCon media did not induce statistically significant changes in SSEA4, Oct4, Sox2 or Nanog expression (Figures $5 \mathrm{a}-\mathrm{C}$ ). Interestingly, it was noted that DiffCon media could replace RA in the MyD88 Peplnh protocol described earlier (data not shown). Collectively, these data indicate that differentiated NTera2 cells secrete factors that self-promote their differentiated state. As MyD88 is a key determinant of the profile of factors secreted by cells generally, and is differentially expressed in these cell states, it is likely that MyD88 is involved in this microenvironment regulation mechanism.

Self-renewing, MyD88 Inhibited, RA-treated and differentiated hECCs secrete specific protein profiles. We next used chemokine-cytokine arrays to characterise some of the factors contained in UndiffCon and DiffCon media as well as media conditioned by 2102Ep cells treated with RA or a small interfering RNA targeting MyD88 ('siMyD88', Supplementary Data 5). Self-renewing NTera2 cells were found to secrete a specific set of proteins that are known regulators of angiogenesis and neurogenesis, and follistatin, a known inhibitor of the Activin signalling (Table 1, Supplementary Data 5). Treatment of 2102Ep cells with either RA or siMyD88 resulted in specific secretion of different sets of angiogenesis regulators (Table 1, Supplementary Data 5). Assessment of DiffCon media indicated secretion of a large set of factors including known regulators of neurogenesis, TGF- $\beta$, Activin and BMP signalling, and immunity (Table 1 , Supplementary Data 5). Together, this indicates a mechanism where hECC differentiation is associated with activation of angiogenic and neurogenic differentiation as well as all three branches of TGF- $\beta$ Superfamily signalling. We have shown above that these are sufficient to differentiate NTera2 cells.

Loss of MyD88 facilitates upregulation of HOX genes and activation of RA Signalling. We next characterised the MyD88 molecular mechanism using whole-genome gene expression array analysis. Initially, 2102Ep cells treated with MyD88 Peplnh or control Peplnh \pm RA were assessed to establish events regulated by RA or loss of MyD88 specifically. Subsequently, differentiated SSEA4 ${ }^{\mathrm{Neg}}$ cells isolated at the end of the 12 day MyD88 Peplnh +RA protocol 

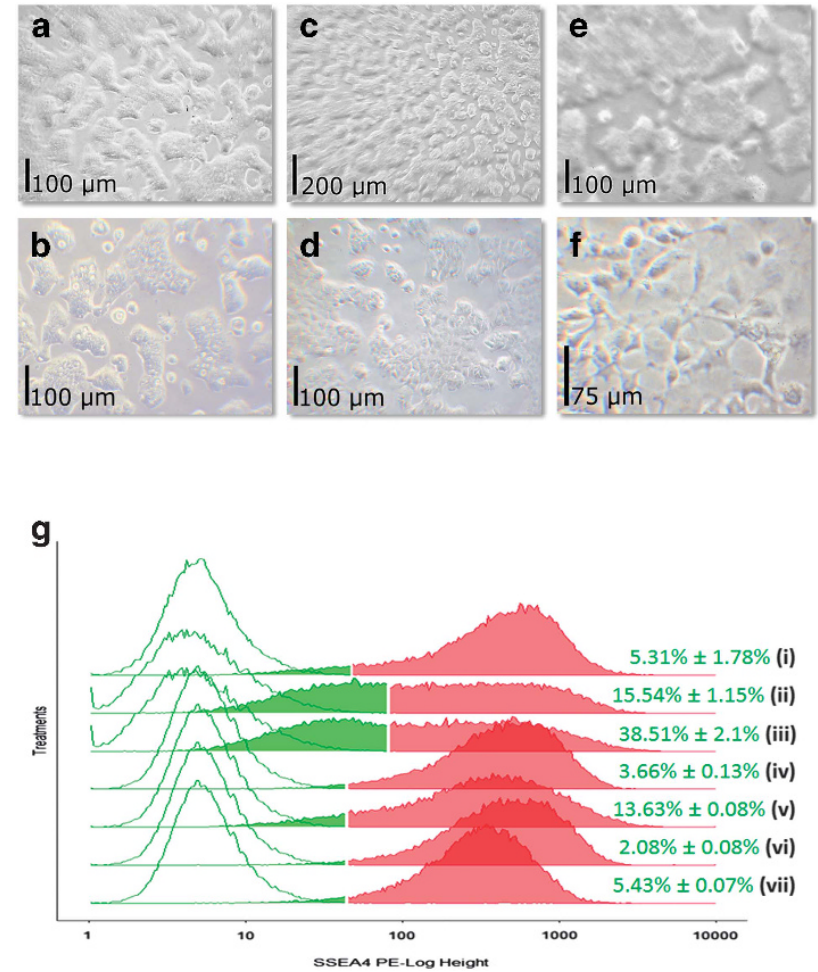
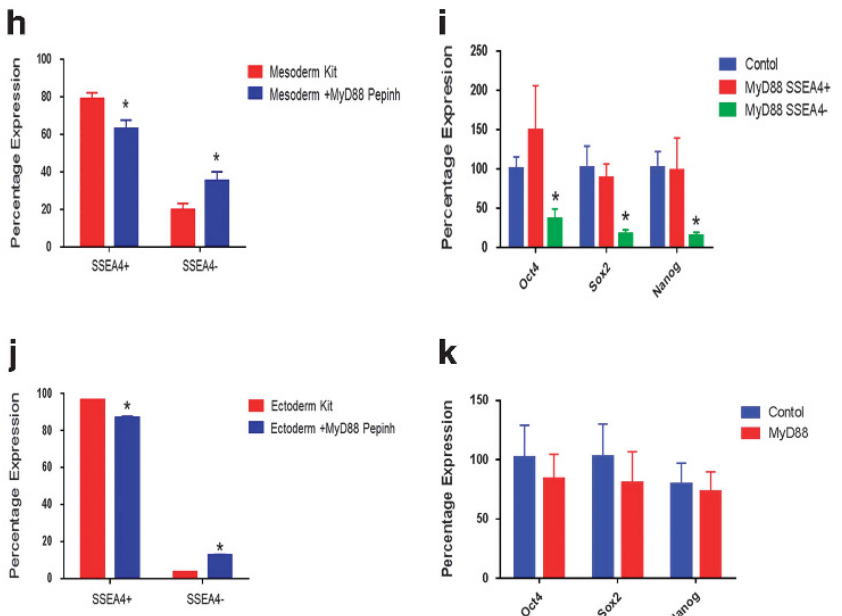

k
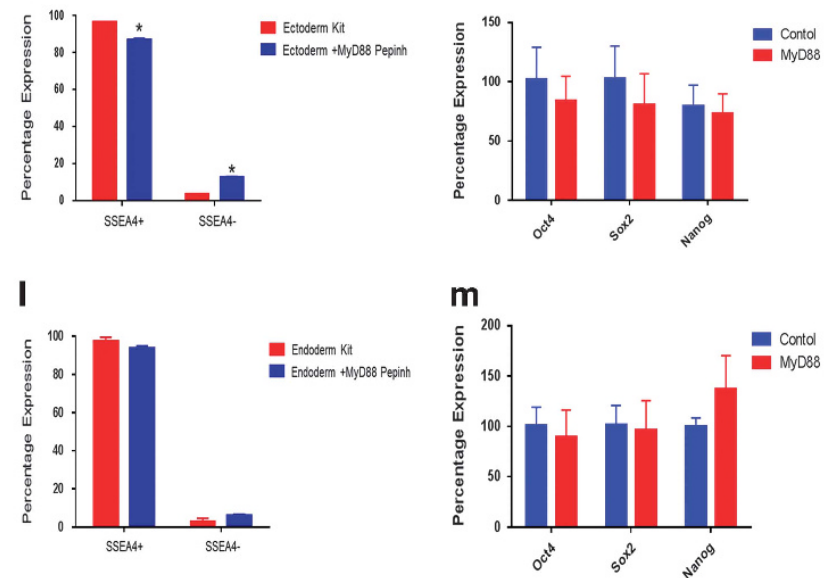

Figure 4 A potential role for MyD88 in mesodermal differentiation of 2102Ep cells. 2102Ep cells were treated with specific endoderm, mesoderm and ectoderm differentiation kits to assess whether the MyD88 mechanism was RA-specific. Morphology images show 2102Ep cells stimulated to differentiate down endoderm (a, b), ectoderm (c, $\mathbf{d}$ ) or mesoderm $(\mathbf{e}, \mathbf{f})$ lineages using specific differentiation kits following treatment with (d-f) or without (a-c) MyD88 Peplnh. Differentiation was only apparent in cells treated with the MyD88 Peplnh and then a mesodermal differentiation protocol (f). Cells were subsequently assessed for differentiation status using SSEA4 flow cytometry and Oct4-Sox2-Nanog qPCR expression levels $(\mathbf{g}-\mathbf{m})$. (g) Open lines represent negative staining controls for each sample. Filled lines represent the stained sample. Green represents the SSEA4 ${ }^{\text {Neg }}$ cells and red the SSEA4 ${ }^{\text {Pos }}$ cells, as determined by the internal negative staining controls (i: untreated control, ii: mesoderm kit only, iii: MyD88 Peplnh+mesoderm kit, iv: ectoderm kit only, v: MyD88 PepInh+ectoderm kit, vi: endoderm kit only, vii: MyD88 Peplnh+endoderm kit). This analysis indicated that 2102Ep cells treated with MyD88 Peplnh+mesoderm protocol produce a population of SSEA4 ${ }^{\text {Neg }}$ cells (a-iii: $\left.38.51 \pm 2.1 \%\right)$ that was significantly larger $(\mathbf{h})$ than observed in control cells $(\mathrm{a}-\mathrm{ii}: 15.54 \pm 1.15 \%)$. Post-FACS isolation qPCR analysis of Oct4-Sox2-Nanog confirmed that these were differentiating cells (i). Cells treated with MyD88+ectoderm protocol produced a significant (a-iv: $3.66 \pm 0.13 \%$; a-v: $13.63 \pm 0.08 \%$; j statistical analysis, $\mathbf{k}$ : qPCR) SSEA4 ${ }^{\mathrm{Neg}}$ population that was insufficient in size to isolate by FACS for qPCR analysis (k). Treatment of cells with the endoderm protocol did not result in differentiation (a-vi: $2.08 \pm 0.08 \%$; a-vii: $5.43 \pm 0.07 \%$; l: statistical analysis, m: qPCR). Morphology data indicate a potential role for MyD88 in mesodermal differentiation. ${ }^{*} P$-value $<0.05$

were assessed to establish downstream events. All samples, including SSEA4 ${ }^{\mathrm{Pos}}$ and MyD88 PepInh+RA controls, passed quality controls, separated well and showed strong clustering (Figures 6a and b, full genelists in Supplementary Data 6). Confirming differentiation, SSEA $4{ }^{\mathrm{Neg}}$ cells were found to downregulate pluripotency markers (Oct4, Sox2, Nanog, Alpl, Gdf3, Tdgf1, Tert, Esrrb, Myc, Sall4, Thap11, Utf1 and Zfp42) and upregulate differentiation markers (Mesoderm: Hand1 and Msx1, Definitive Endoderm: Gata6, Cardiac Progenitor: Isl1, Early Smooth Muscle: Acta2, Haematopoietic Stem Cell/ Early Endothelial Cells: Gata2, Mesenchymal Stem Cells: CD44 and Eng) compared with SSEA4 ${ }^{\text {pos }}$ cells. In overview, while distinct sets of genes were altered by treatment with MyD88 Peplnh or RA, differentiation requires their combination (Figure 6c). MyD88 inhibition resulted in alteration of 414 genes (119 Up, 295 Down) related to differentiation/embryonic development (Supplementary Data 6\&7). Analysis of molecular relationships via the online tool DAVID ${ }^{24}$ highlighted the primary effect of MyD88 inhibition as upregulation of four HOX genes and inhibition of five Olfactory Receptor (OR) genes and seven taste receptor type 2 (T2Rs) genes (Table 2). These sensory receptor genes have, in recent years, been shown to be expressed in nonsensory tissue such as testis, where they clearly perform a non-sensory role. ${ }^{25,26}$ The relationship between RA treatment and the alteration of HOX and OR genes is long established. However, this is the first description of their involvement in a MyD88-dependent pre-RA treatment mechanism. MyD88 inhibition alone did not result in substantial changes in Oct4, Sox2 or Nanog, RA or TLR Signalling gene expression, which suggests that MyD88 inhibited cells are in a pluripotent/nullipotent state in which MyD88-independent TLR signalling has not been activated. Comparison of control Peplnh versus control Peplnh+RA treatment indicated that $R A$ resulted in alteration of only 71 genes (47 Up, 24 Down) including upregulation of RA Signalling (RarB, Crabp1) and additional $\operatorname{HOX}(A 1, A 2, A 4$, B1) genes (Table 2, Figure 6c).

Analysis of the 3491 genes (1697 Up, 1794 Down) altered in SSEA $4^{\mathrm{Neg}}$ cells (compared with MyD88 Peplnh-treated cells) highlighted loss of Oct4, Sox2 and Nanog expression as well as upregulation of additional RA Signalling (Stra6, Crabp2, RarA and further upregulation of RarB) and $\mathrm{HOX}$ (B3, C8, C13 and further upregulation of $\mathrm{A} 1$ and $\mathrm{A} 2$ ) genes, all of which are 
a

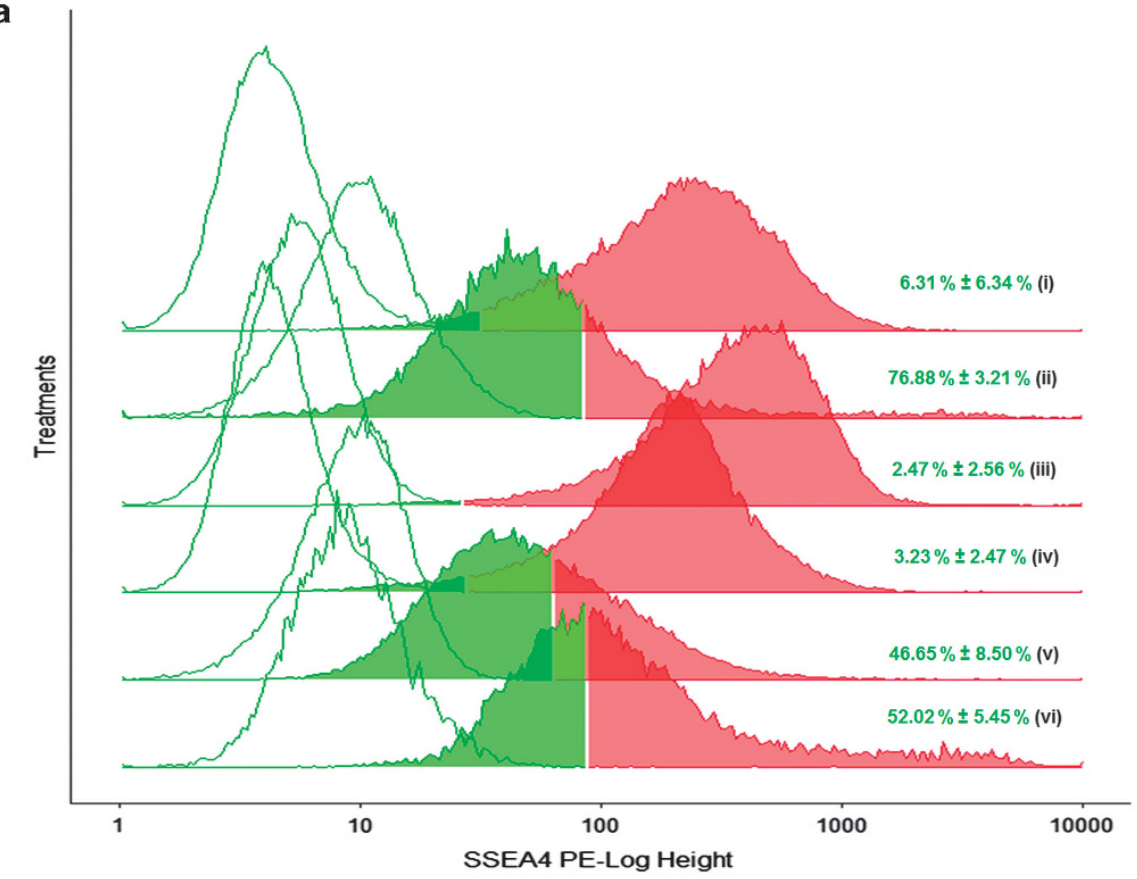

b

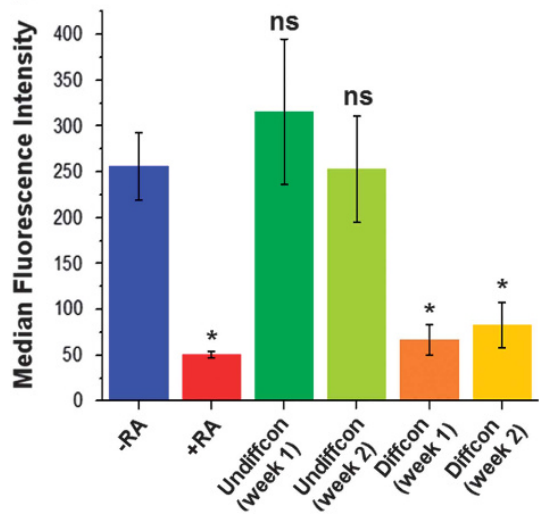

C

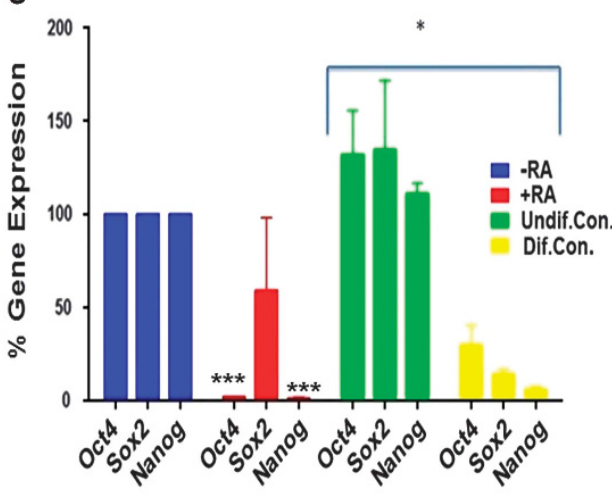

Figure 5 Differentiated NTera2 cells secrete specific protein profiles sufficient to promote differentiation. Undifferentiated NTera2 cells were grown for 7 days in media conditioned by (collected for 7 or 14 days) undifferentiated (UndiffCon) or pre-differentiated (DiffCon) NTera2 cells, after which SSEA4 expression levels were assessed using flow cytometry (a). Open lines represent negative staining controls for each sample. Filled lines represent the stained sample. Green represents the SSEA4 ${ }^{\text {Neg }}$ cells and red the SSEA4 ${ }^{\text {Pos }}$ cells, as determined by internal negative staining controls (a-i: - RA \& a-ii: +RA controls). Treatment with DiffCon media (collected for 7 (v) or 14 (vi) days) was found to reduce SSEA4 expression levels, data that were found to be statistically significant (b). This was accompanied by statistically significant decreases in qPCR measured levels of Oct4-Sox2-Nanog expression (c, 7 day DiffCon). In contrast, UndiffCon media (collected for 7 (iii) or 14 (iv) days) had no effect on SSEA4 expression (a, b). No statistically significant changes in qPCR measured levels of Oct4-Sox2-Nanog expression were observed for UndiffCon media (c).These data indicate that differentiated cells secrete factors that promote differentiation. ${ }^{*} P$-value $<0.05 ;{ }^{* *} P$-value $<0.01, \mathrm{~ns}=$ not significant

characteristics of RA-induced differentiation of hECCs (Table 2, Figure 6c). Further mining of the genelist identified several changes indicative of a switch to MyD88-independent TLR Signalling, including downregulation of TIr4 and upregulation of TIr3 and TIr7 (24 fold) expression, as well as upregulation of 16 ISGs and a small number of JAK-STAT signalling genes, none of which are altered in cells treated with MyD88 Peplnh or RA alone (Table 2, Supplementary Data 6). Interestingly, loss of OR and T2R expression following MyD88 inhibition appears to be rescued in SSEA4 ${ }^{\mathrm{Neg}}$ cells. In summary, these data indicate that, in self-renewing cells, MyD88 functions to prevent differentiation by inhibiting and promoting the expression of $\mathrm{HOX}$ and OR/T2R genes respectively. The combination of MyD88 inhibition and RA leads to differentiation of $2102 E p$ cells via activation of RA and MyD88-independent TLR signalling, downregulation of Oct4Sox2-Nanog, and upregulation of HOX genes, as illustrated in Figure 6c.

\section{Discussion}

An improved understanding of the mechanisms that regulate the early differentiation of pluripotent cells is required. In this study, we report that MyD88 is an essential component of hECC pluripotency, which must be downregulated to facilitate RA-induced differentiation. At a molecular level, high MyD88 
Table 1 Selected proteins secreted by self-renewing (UndiffCon media) and differentiated (DiffCon media) NTera2 cells and 2102Ep cells treated with RA or siMyD88

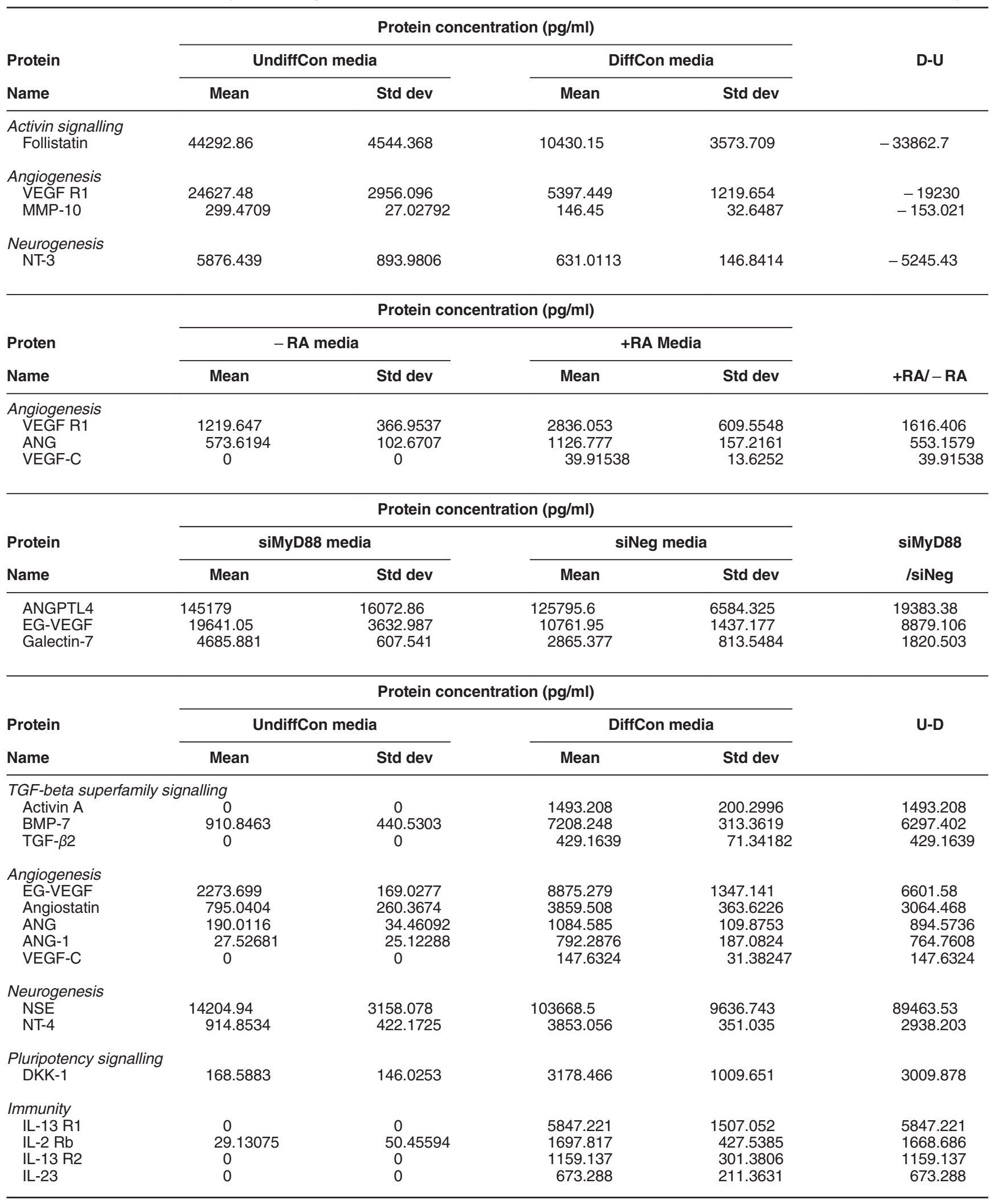




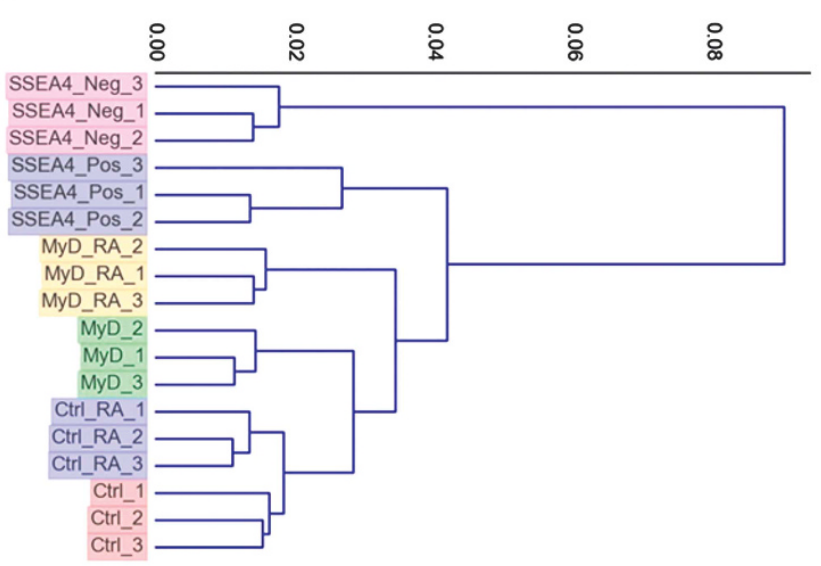

b

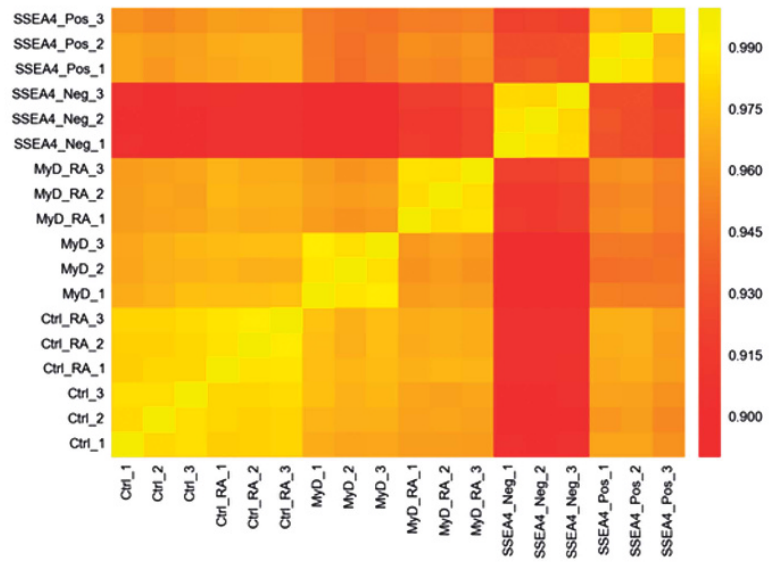

c

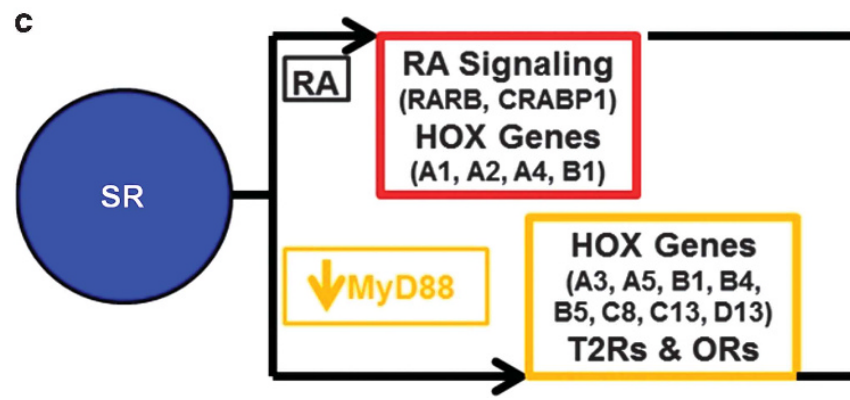

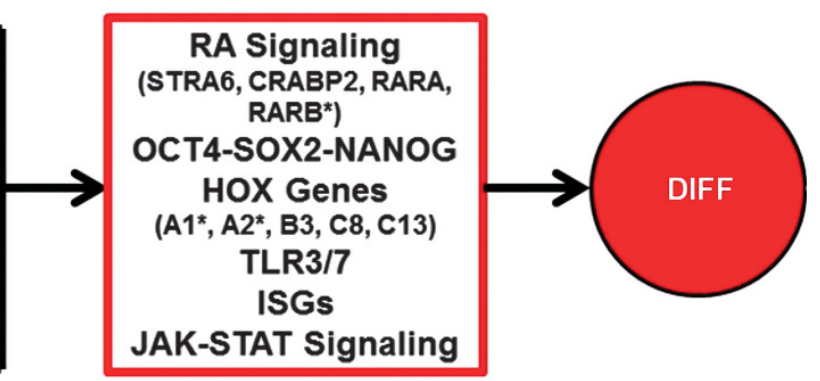

Figure 6 Gene expression array analysis of the MyD88 inhibition facilitated RA differentiation mechanism. Whole-genome gene expression array analysis of the following samples $(n=3)$ was undertaken: control Peplnh-RA (Ctrl), control Peplnh +RA (Ctrl_RA), MyD88 Peplnh -RA (MyD), MyD88 Peplnh +RA (MyD_RA) and post-experiment isolated SSEA4 ${ }^{\text {Pos }}$ (SSEA4Pos) and SSEA4 ${ }^{\mathrm{Neg}}$ (SSEA4Neg) 2102Ep cells. All biological replicates clustered well and all samples separated well (a, b). Selected genelists for MyD88 Peplnh versus control Peplnh, control Peplnh-RA versus control Peplnh+RA, and SSEA4 ${ }^{\text {Neg }}$ versus MyD88 Peplnh comparisons are shown in Table 2 (full genelists in Supplementary Data 6). Together, the data reveal an overall mechanism (c) where RA or MyD88 Peplnh treatment of self-renewing (SR) cells results in the upregulation of selected RA and HOX genes, whereas loss of MyD88 is also associated with downregulation of taste family 2 and olfactory receptor (T2R, OR) genes. RA treatment following MyD88 Peplnh treatment results in activation of RA and MyD88-independent TLR signalling mechanisms as well as further activation of HOX genes. This results in differentiation (Diff), which appears to follow a standard hECC pattern through downregulation of Oct4, Sox2 and Nanog gene expression

expression appears to inhibit functional activation of HOX gene expression to promote the SR state, while its loss facilitates activation of RA signalling, leading to differentiation driven by loss of Oct4-Sox2-Nanog. During RA-induced differentiation, loss of MyD88 forms part of an overall differentiation mechanism that is associated with the secretion of a specific set of proteins, which appears to be due to activation of MyD88-independent TLR signalling. These secreted proteins include known promoters of all three branches of TGF- $\beta$ Superfamily signalling, angiogenesis and neurogenesis, and are sufficient to facilitate differentiation. Although these data require further functional validation, a proposed integrated model of pluripotent hECC RA differentiation is offered in Figure 7.

The pluripotent state is primarily determined by the presence of regulatory growth factors in the niche., 7 The highlighting of MyD88 in this study, which determines the profile of many proteins secreted by the cell, matches this model. In particular, pluripotency/differentiation is regulated by all three branches of TGF- $\beta$ Superfamily signalling. ${ }^{7,3}$ As such, our demonstration that MyD88 regulates hECC pluripotency and hECC secretion of regulators of all three branches of TGF$\beta$ Superfamily signalling also matches this model. Specifically, hECCs appear to use self-secreted proteins to inhibit Activin signalling in the SR state before promoting differentiation though activation of BMP and TGF- $\beta$ signalling. Continuing these similarities, it is notable that a SR state that appears to be primed for RA (neural lineage) differentiation can be generated through MyD88 inhibition in 2102Ep cells. This is an interesting parallel to the primed state model of ESC pluripotency. Although it is important to note that this is a malignant, and thus clearly mutated, model of pluripotency, the mechanisms revealed here, or similar non-malignant mechanisms, may prove useful for exploitation in the hESC or iPSC field. For example, it may be possible to exploit the MyD88 mechanism described here, or similar mechanisms, to generate primed pluripotent cells whose differentiation can be better controlled. However, it is known that there are many key differences between murine and human pluripotency: we note that MyD88 knockout mice appear to have no non-immunity related defects, ${ }^{20,27-29}$ suggesting that MyD88 is not an essential regulator of the mammalian SR state. As such, collectively our data indicates that MyD88 is a redundant regulator of mammalian pluripotency, specific regulator of human pluripotency, or specific regulator of human malignant pluripotency. As inhibition/knockdown of MyD88 has not been performed in hESCs to our knowledge, these questions remain open. 
Table 2 Selected genes expressed by 2102Ep cells treated with MyD88 compared with control Peplnh, control Peplnh compared with control Peplnh $+\mathrm{RA}$, and MyD88 Peplnh compared with SSEA4 ${ }^{\mathrm{Neg}}$ cells

\begin{tabular}{|c|c|c|}
\hline \multicolumn{3}{|c|}{ SSEA4Neg versus MyD88 Peplnh } \\
\hline Gene name & Fold change & $P$-value \\
\hline \multicolumn{3}{|l|}{ Pluripotency } \\
\hline Nanog & -7.2 & $1.89 E-05$ \\
\hline Sox2 & -7.2 & $2.89 E-06$ \\
\hline Oct4 & -1.8 & 0.01 \\
\hline \multicolumn{3}{|c|}{ Toll-like receptors } \\
\hline TLR7 & 24 & $6.20 \mathrm{E}-07$ \\
\hline TLR3 & 3.2 & $1.57 \mathrm{E}-05$ \\
\hline TLR4 & -2.5 & 0.00055 \\
\hline \multicolumn{3}{|l|}{ RA signalling } \\
\hline STRA6 & 2.3 & 0.00017 \\
\hline CRABP2 & 2.2 & 0.00015 \\
\hline RARB & 8.8 & $1.68-\mathrm{E} 06$ \\
\hline RARA & 2 & 0.00038 \\
\hline RARRES3 & 2.3 & $6.60 E-06$ \\
\hline RAI 14 & 2.6 & $3.60 E-05$ \\
\hline \multicolumn{3}{|l|}{ HOX genes } \\
\hline HOXA1 & 6.8 & $3.08 \mathrm{E}-06$ \\
\hline HOXA2 & 3.7 & 2.77E - 05 \\
\hline HOXАЗ & 2.4 & 0.0015 \\
\hline HOXA5 & 3.4 & 1.77E-05 \\
\hline HOXB1 & 4.2 & $9.53 E-06$ \\
\hline HOXB3 & 7.2 & $2.71 \mathrm{E}-06$ \\
\hline HOXB4 & 4.9 & $2.93 E-05$ \\
\hline HOXB5 & 3.3 & 3.04E - 05 \\
\hline HOXC8 & 2.4 & 0.0023 \\
\hline $\mathrm{HOXC13}$ & 2.9 & 0.00015 \\
\hline \multicolumn{3}{|c|}{ Interferon-stimulated genes (ISGs) } \\
\hline IFI44L & -85.4043618 & $2.08 \mathrm{E}-11$ \\
\hline IFIT3 & -35.59805019 & $1.55 \mathrm{E}-09$ \\
\hline $\mathrm{IFIH} 1$ & -31.50036308 & $1.71 \mathrm{E}-09$ \\
\hline IFI44 & -25.502419 & $3.92 E-09$ \\
\hline IFI6 & -25.27572766 & $2.85 E-10$ \\
\hline IFIT1 & -17.39771836 & $2.24 \mathrm{E}-09$ \\
\hline IFIT2 & -16.18933034 & $1.87 \mathrm{E}-09$ \\
\hline IFI27 & -11.32773296 & $1.29 E-07$ \\
\hline IRF9 & -8.488694247 & $1.08 \mathrm{E}-08$ \\
\hline GBP2 & -8.195717684 & $1.21 \mathrm{E}-07$ \\
\hline IFI35 & -4.23411297 & $2.23 E-07$ \\
\hline IRF1 & -3.98565266 & $1.64 \mathrm{E}-07$ \\
\hline IFIT5 & -3.970552695 & $1.97 \mathrm{E}-06$ \\
\hline IRF2BP2 & -2.692853863 & $2.18 \mathrm{E}-06$ \\
\hline IRF7 & -2.120580336 & $1.9 \mathrm{E}-05$ \\
\hline IFI16 & -2.111790274 & $1.11 \mathrm{E}-05$ \\
\hline \multicolumn{3}{|c|}{ JAK-STAT signalling } \\
\hline STAT1 & 4.7 & $3.93 E-08$ \\
\hline
\end{tabular}

Our data feed into an on-going discussion about the relationship between TLR signalling and pluripotency. ESCs express TLRs and can respond to microbial products, a response that can enhance myeloid-lineage differentiation. ${ }^{30}$ TLR ligands are reportedly secreted by murine ESCs (mESCs) ${ }^{31}$ but not hESCs. ${ }^{32}$ There is also clearly an overlap between TLR signalling and RA. For example, RA-Inducible gene 1 is an established viral pattern recognition receptor in innate immunity, ${ }^{33}$ which can be stimulated by bacterial wall component lipopolysaccharide (LPS), ${ }^{34}$ is necessary for bacterial phagocytosis in macrophages, and is required for RA differentiation of myeloid cells. ${ }^{35} \mathrm{MyD} 8^{17}$ and $\mathrm{Toll}^{36}$ were originally identified as a modulator of myeloid cell differentiation and a developmental regulator in Drosophila, respectively, roles apparently distinct from immunity. MyD88 has been shown to be expressed and functional in some mESC lines ${ }^{37}$ as well as, for example, mesenchymal ${ }^{38}$ and haematopoietic $^{39}$ adult stem cells. In the MyD88 mechanism we have described here, upregulation of TLR3 may result in chromatin remodelling to facilitate changes in expression of key differentiation genes, in a similar manner to that underlying increased iPS efficiency in TLR3 expressing cells. ${ }^{40,41}$ In addition, there is no clear evidence that innate immunity is required by or functional in such early stages of embryogenesis (inner cell mass cells within the blastocyst). As such, our data support a broader view of the role of TLR signalling, where the pathway can respond to different stimuli at different developmental stages. In this regard, TLR signalling may serve to regulate the stem cell or cancer stem cell (CSC) niche through the regulated secretion of specific proteins by the cell.

Tumour-initiation is a property of self-renewing CSCs such as hECCs, which is lost during differentiation. ${ }^{42}$ In the developing tumour, CSCs that persist in the SR state while other CSCs differentiate are, therefore, clear clinical targets. Global targeting of CSCs via forced-differentiation treatments using drugs such as RA has been largely inefficient in the clinic, apart from in specific patients with specific malignancies, ${ }^{43-45}$ which indicates the importance of better understanding CSC differentiation resistance. Although the mechanisms through which certain CSCs resist differentiation are unknown, it is likely to involve regulation of growth factors in specific SR niches within the tumour. For example, in ovarian CSCs, MyD88-dependent TLR signalling has been shown to create a microenvironment that facilitates selfrenewal. ${ }^{46}$ As such, it is interesting that we have highlighted a role for MyD88, a key determinant of growth factor secretion into the niche, in differentiation resistance. We have shown that hECC nullipotency, an extreme model of differentiation resistance, is owing to MyD88 acting as a 'Differentiation Gatekeeper' that must be compromised to facilitate differentiation. We have previously shown that 2102Ep nullipotency can be surpassed by forced-differentiation via knockdown of Sox2. ${ }^{47}$ As such, it appears that maintained MyD88 expression in 2102Ep cells is owing to a defect in the MyD88 downregulation mechanism, the search for which will continue. It is possible that similar differentiation gatekeepers exist in other malignancies. If so, our data suggest that a two-tier strategy involving a differentiation morphogen such as RA in combination with targeting of a specific differentiation regulator may be more successful than treatment with a single agent. Although the MyD88 Peplnh approach employed here was highly efficient, it is not clear whether MyD88, as a key regulator of immunity, could be inhibited in the clinic without detrimental side effects for the patient. However, if essential differentiation regulators can be identified in other malignancies, their targeting may synergistically enhance the effects of differentiation treatments.

\section{Materials and Methods}

Cell culture, transfections and differentiation protocols. Cell culture, siRNA transfection and RA differentiation were performed as previously described. ${ }^{47,48}$ MyD88 overexpression was achieved by transfection of a constitutive expression plasmid (IOH9958-pDEST26, IMAgene, Berlin, Germany) for 3 days. 


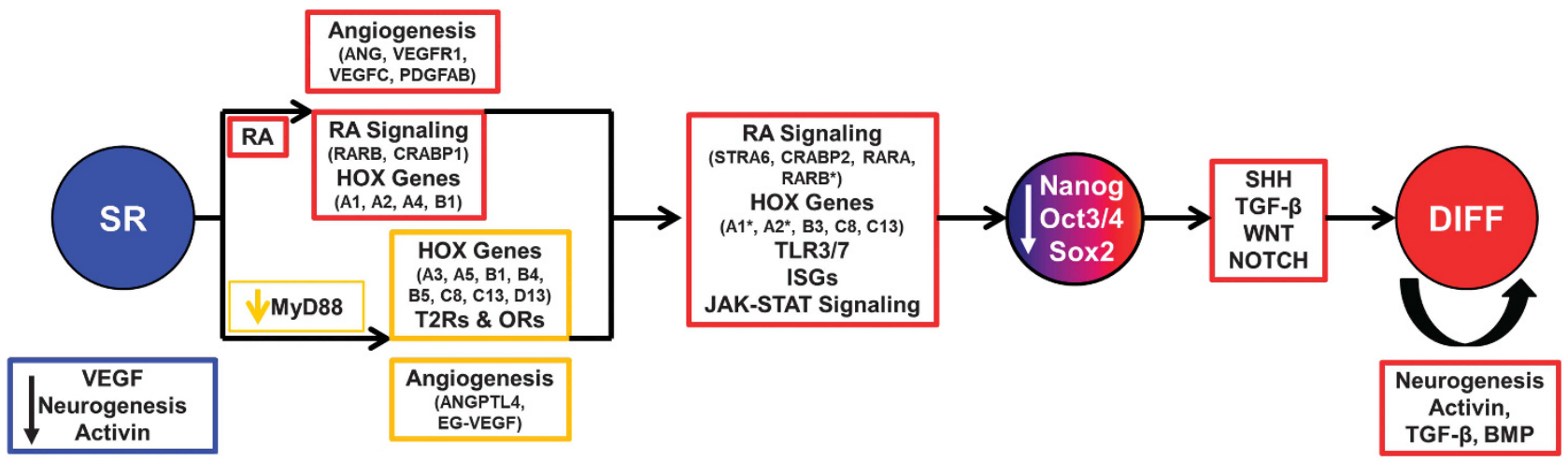

Figure 7 Overall Mechanism: Loss of MyD88 facilitates RA-Induced Differentiation of hECCs. NTera2 pluripotent, and 2102Ep hECCs were assessed to characterise the role of MyD88 in the RA differentiation mechanism. The arrows in the centre indicate the molecular changes observed in 2102Ep cells treated with RA or a MyD88 Peplnh. Loss of MyD88 activates expression of specific Hox genes and downregulates the expression of specific Type 2 Family Taste Receptor (T2R) and Olfactory Receptor (OR) genes (Yellow box), whereas RA treatment activates the expression of specific RA signalling and Hox genes (Red box). Although neither is sufficient for differentiation of $h E C C s$, their combination results in the activation of RA and MyD88-Independent TLR Signalling, and further expression of Hox genes. This results in differentiation through standard downregulation of Oct4-Sox2-Nanog mechanisms, which result in upregulation of stemness signalling pathways such as Sonic Hedgehog (SHH), TGF- $\beta$, Wnt and Notch (Supplementary Data 1). In parallel, self-renewing (SR) and differentiated (DIFF) NTera2 cells were found to secrete specific protein profiles: this differentiated cell secretion profile was found to be sufficient to promote differentiation. When characterised using protein array analysis of conditioned media, SR cells were found to secrete several factors including inhibitors of Angiogenesis, Neurogenesis and Activin Signalling (Blue box). During early differentiation, both loss of MyD88 (Yellow Box) and RA (Red box) treatment result in secretion of a group of angiogenesis regulators. Finally, differentiated NTera2 cells continue secretion of these angiogenesis regulators as well as Activin, TGF- $\beta$, SHH and Neurogenic factors

MyD88 inhibition was achieved by addition of $5 \mu \mathrm{M}$ MyD88 Peptide Inhibitor (Peplnh, Invivogen, San Diego, CA, USA) or the supplied control Peplnh to 5056000 (24-well plate) or 168000 (six-well plate) cells/well. After an initial $6 \mathrm{~h}$ treatment, Peplnh was refreshed and cells cultured overnight, after which RA $\left(10^{-5} \mathrm{M}\right)$ and Peplnh were added together with media refreshed daily. Lineage differentiation assays were carried out similarly, with the addition of growth factor solutions supplied in specific StemXvivo Mesoderm, Ectoderm or Endoerm kits (R\&D Systems, Abingdon, UK) instead of RA, and using manufacturer's instructions. Specific medias were refreshed daily for 6 days.

Quantitative ELISAs. MyD88 inhibition was assayed by treating 56000 cells with MyD88 or control Peplnh for $24 \mathrm{~h}$ and then with IL-1 $\beta$ (30 ng/ml, InvivoGen), which can only act MyD88-dependently, for $30 \mathrm{~min}$. Activation of MyD88-dependent signalling was detected as the presence of phosphorylated-I- $\kappa \mathrm{B} \alpha$ using the 'PhosphoTracer $\mathrm{I}-\kappa \mathrm{B} \alpha$ Total ELISA' kit (Abcam, Cambridge, UK) as per manufacturer's instructions. Alkaline Phosphatase expression was quantified using the 'Quantitative Alkaline Phosphatase ES Characterisation Kit' (Millipore, Nottingham, UK) as per manufacturer's instructions.

Conditioned media work. UndiffCon media was prepared by collecting media from NTera2 cells daily for 7 days. For DiffCon media, NTera2 cells were RA differentiated for 7 days. These differentiated cells were then cultured for a further 7 days in -RA media, which was collected and refreshed daily. Conditioned media was pooled and filtered using a $0.2 \mu \mathrm{m}$ polyethersulfone filter. For conditioned media treatments, $2 \mathrm{ml}$ of conditioned media was added to 180000 cells in six-well plates and replaced daily.

Flow cytometry/FACS. For each sample, after dissociation using EDTA (1 mM), 1 million cells were assayed with PE-conjugated mouse IgG3 anti-SSEA4 antibody (MC-813-70, R\&D Systems), using PE-conjugated mouse IgG3 (133316, R\&D Systems) and unstained samples as isotype and autoflourescence, respectively. Flow cytometry was performed on a Cyan ADP Flow Cytometer (Beckman Coulter, High Wycombe, UK). Samples were excited using a $488 \mathrm{~nm}$ laser and detected between $575 / 25 \mathrm{~nm}$. Doublets were excluded using the pulse width parameter and dead cells were excluded using propidium iodide staining (Invitrogen; Excited $=488 \mathrm{~nm}$; detected $=680 / 30 \mathrm{~nm}$ ). FACS was performed using the MoFlo XDP (Beckman Coulter) and FACSAria Fusion cell sorters (BD Bioscience, Oxford, UK). Flow cytometry was carried out at the TCD facilities in the
Institute of Molecular Medicine (IMM), St James's Hospital, Dublin 8 and the Trinity Biomedical Sciences Institute (TBSI), Pearse Street, Dublin 8.

Western blot, qPCR, gene and protein array analysis. Western blot analysis was carried out as previously described 47; Antibodies; MyD88: \#4283 Cell Signalling Technologies, Gapdh: Ab8245, Abcam). qPCR was carried out as previously described. ${ }^{12}$ RNA was isolated using the mirVANA kit (Ambion, Waltham, MA, USA). Three- day differentiation experiment gene expression array analysis was carried out using HGU133 Plus2 GeneChips. A total of $1 \mu \mathrm{g}$ RNA was prepared using the 'One-Cycle Eukaryotic Target Labelling' protocol and assayed on HGU133 Plus2 GeneChips (All Affymetrix, Waltham, MA, USA) as per manufacturer's instructions. All other gene expression array analysis was carried out using Affymetrix GeneChip HuGene 2.0 ST arrays. Final $150 \mathrm{ng}$ samples were prepared using the 'WT Plus reagent Kit', and prepared samples fragmented and labelled using the 'WT Labelling Kit' (Affymetrix) as per manufacturer's instructions. Post hybridisation, all samples were washed using a fluidics station 450 and scanned using a GeneChip Scanner 3000 (both Affymetrix) as per manufacturer's instructions. Pre-processing and analysis of gene expression array data was performed as described in Supplementary Methods. For protein arrays, conditioned media samples were concentrated using Amicon Ultracel $3 \mathrm{kDa}$ spin filters (Millipore) and assayed using Quantibody Human Chemokine and Cytokine arrays (RayBioTech, Norcross, GA, USA) as per manufacturer's instructions.

Statistical analysis. Statistical analysis of flow cytometry, protein array and qPCR data was carried out using the student's two-tailed t-test. Data is presented as the mean of three biological replicates \pm S.E.M. GraphPad prism 6 was used to generate $P$-values.

\section{Conflict of Interest}

The authors declare no conflict of interest.

Acknowledgements. This work was primarily supported by funding from The Irish Cancer Society (JOL, MG, AC, Grant: CRP08OLE), The Libyan Education Authority (GS \& SE) and the European Union (7th Framework: PASCA, JOL, MG, BF, $\mathrm{HK}, \mathrm{VMcE}$ ). Work was also carried out by researchers supported by Science Foundation Ireland (JOL; IBJ 13/SIRG/2128), the Health Research Board (HRB, Ireland. JOL) and the Emer Casey Foundation (BF). We would like to thank Professor Peter Andrews, University of Sheffield, for his technical advice and support 
throughout the study, and Dr Paul J Gokhale (University of Sheffield), Dr Nobue Itasaki (University of Bristol) and Dr Vincent Kelly (TCD) for their technical input during data analysis. We thank Dr Eamon Breen (IMM, TCD) and Dr Barry Moran (TBSI, TCD) for their help with flow cytometry. Funding for the flow cytometry facility (TBSI, TCD) was provided by Science Foundation Ireland (Grant 12/ $\mathrm{RI} / 2340(7))$.

1. Evans MJ, Kaufman MH. Establishment in culture of pluripotent cells from mouse embryos. Nature 1981; 292: 154-156.

2. Thomson JA, Itskovitz-Eldor J, Shapiro SS, Waknitz MA, Swiergiel JJ, Marshall VS et al. Embryonic stem cell lines derived from human blastocysts. Science 1998; 282: 1145-1147.

3. De Los Angeles A, Ferrari F, Xi R, Fujiwara Y, Benvensity N, Deng H et al. Hallmarks of pluripotency. Nature 2015; 525: 469-479.

4. Martello G, Smith A. The nature of embryonic stem cells. Annu Rev Cell Dev Biol 2014; 30 $647-675$.

5. Dodsworth BT, Flynn R, Cowley SA. The current state of naïve human pluripotency. Stem Cells 2015; 33: 3181-3186.

6. Guo G, von Meyenn F, Santos F, Chen Y, Reik W, Bertone P et al. Naïve pluripotent stem cells derived directly from isolated cells of the human inner cell mass. Stem Cell Rep 2016; 6 : 437-446.

7. Silva J, Smith A. Capturing pluripotency. Cell 2008; 132: 532-538.

8. Andrews PW. From teratocarcinomas to embryonic stem cells. Philos Trans $R$ Soc Lond $B$ Biol Sci 2002; 357: 405-417.

9. Andrews PW, Martin MM, Bahrami AR, Damjanov I, Gokhale P, Draper JS. Embryonic stem (ES) cells and embryonal carcinoma (EC) cells: opposite sides of the same coin. Biochem Soc Trans 2005; 33: 1526-1530.

10. Josephson R, Ording CJ, Liu Y, Shin S, Lakshmipathy U, Toumadje A et al. Quantification of embryonal carcinoma 2012Ep as a reference for human embryonic stem cell research. Stem Cells 2007; 25: 437-446.

11. Evans MJ. Discovering pluripotency: 30 years of mouse embryonic stem cells. Nat Rev Mol Cell Biol 2011; 12: 680-686.

12. D'Adhemar CJ, Spillane CD, Gallagher MF, Bates M, Costello KM, Barry-O'Crowley J et al. The MyD88+ phenotype is an adverse prognostic factor in epithelial ovarian cancer. PLOS ONE 2014; 9: e100816.

13. Chambon P. A decade of molecular biology of retinoic acid receptors. FASEB J 1996; 10 : 949-954.

14. Mallo M, Alonso CR. The regulation of Hox gene expression during animal development. Development 2013; 140: 3951-3963.

15. Das BC, Thapa P, Karki R, Das S, Mahapatra S, Liu TC et al. 2014 Retinoic acid signalling pathways in development and diseases. Bioorg Med Chem 2014 22: 673-683.

16. Cunningham TJ, Deuster $G$. Mechanisms of retinoic acid signalling and its roles in organ and limb development. Nat Rev Mol Cell Biol 2015; 16: 110-123.

17. Lord KA, Hoffman-Liebermann B, Liebermann DA. Complexity of the immediate early response of myeloid cells to terminal differentiation and growth arrest includes ICAM-1, JunB and histone variants. Oncogene 1990; 5: 387-396.

18. Liebermann DA, Hoffman B. Myeloid differentiation (MyD) primary response genes in hematopoiesis. Oncogene 2002; 21: 3391-3402.

19. O'Neill LA, Golenbock D, Bowie AG. The history of Toll-lie receptors-redifining innate immunity. Nat Rev Immunol 2013; 13: 453-460.

20. Kawai T, Adachi O, Ogawa T, Takeda K, Akira S. Unresponsiveness of MyD88-defieient mice to endotoxin. Immunity 1999; 11: 115-122.

21. Lu YC, Yeh WC, Ohashi PS. LPS/TLR4 signal transduction pathway. Cytokine 2008; 42 145-151.

22. Torres J, Prieto J, Durupt FC, Broad S, Watt FM. Efficient differentiation of embryonic stem cells into mesodermal precursors by BMP, retinoic acid and notch signalling. PLOS ONE 2012; 7: e36405.

23. Oeda S, Hayashi $\mathrm{Y}, \mathrm{Chan} \mathrm{T}$, Takasato M, Ahihara $\mathrm{Y}$, Okabayashi $\mathrm{K}$ et al. Induction of intermediate mesoderm by retinoic acid receptor signalling from differentiating mouse embryonic stem cells. Int J Dev Biol 2013; 57: 383-389.

24. Huang, da W, Sherman BT, Lempicki RA. Systematic and integrative analysis of large gene lists using DAVID bioinformatics resources. Nat Protoc 2009; 4: 44-57.

25. Kaupp UP. Olfactory signalling in vertebrates and insects: differences and commonalities. Nat Rev Neurosci 2010; 11: 188-200.
26. Li F. Taste perception: from the tongue to the testis. Mol Hum Reprod 2013; 19: 349-360.

27. Aldachi O, Kawai T, Takeda K, Matsumoto M, Tsutsui H, Sakagami M et al. Targeted disruption of the MyD88 gene results in loss of IL-1 and IL-18-mediated function. Immunity 1998; 9: 143-150.

28. Salcedo R, Worschech A, Cardone M, Jones Y, Gyulai Z, Dai RM et al. MyD88-mediated signalling prevents development of adenocarcinomas of the colon: role of interleukin 18. J Exp Med 2010; 207: 1625-1636.

29. Bezerra DaSilva RA, Nelson-Filho P, Lucisano MP, De Rossi A, de Queiroz AM, Bezerra da Silva LA. MyD88 knockout mice develop initial enlarged periapical lesions with increased numbers of neutrophils. Int Endod J 2014; 47: 675-686.

30. Lee SH, Hong B, Sharabi A, Huang XF, Chen SY. Embryonic stem cells and mammary luminal progenitors directly sense and respond to microbial products. Stem Cells 2009; 27: 1604-1615.

31. Guo Y, Graham-Evans B, Broxmeyer HE. Murine embryonic stem cells secrete cytokines/ growth modulators that enhance cell survivalanti-apoptosis and stimulate colony formation of murine hematopoietic progenitor cells. Stem Cells 2006; 24: 850-856.

32. Sarkar P, Randall SM, Muddiman DC, Rao BM. Targeted proteomics of the secretory pathway reveals the secretome of mouse embryonic fibroblasts and human embryonic stem cells. Mol Cell Proteomics 2012; 11: 1229-1839.

33. Matsumiya T, Stafforini DM. Function and regulation of retinoic acid-inducible gene-1. Crit Rev Immunol 2010; 30: 489-513.

34. Imaizumi T, Aratani S, Nakajima T, Carlson M, Matsumiya T, Tanji K et al. Retinoic acidinducible gene-l is induced in endothelial cells by LPS and regulates expression of COX-2. Biochem Biophys Res Commun 2002; 292: 274-179.

35. Kong L, Sun L, Zhang H, Liu Q, Liu Y, Qin L et al. An essential role for RIG-I in toll-like receptor-stimulated phagocytosis. Cell Host Microbe 2009; 6: 150-161.

36. Anderson KV, Jurgens G, Nusslein-Volhard C. Establishment of dorsal-ventral polarity in the drosophila embryo: genetic studies on the role of the Toll gene product. Cell 1985; 42: 779-789.

37. Taylor T, Kim Y-J, Ou X, Derbigny W, Broxmeyer HE. Toll-Like Receptor 2 mediates proliferation, survival, NF- $\mathrm{kB}$ translocation, and cytokine mRNA expression in LIFmaintained mouse embryonic stem cells. Stem Cell Dev 2010; 19: 1333-1341.

38. Martino MM, Maruyama K, Kuhn GA, Satoh T, Takeuchi O, Muller R et al. Inhibition of IL-1R1/MyD88 signaling promotes mesenchymal stem cell-drive tissue generation. Nat Comm 2016; 7: 11051.

39. Nagai $\mathrm{Y}$, Garrett KP, Ohta S, Bahrun U, Kouro T, Akira A et al. Toll-like receptors on hematopoietic progenitor cells stimulate innate immune system replenishment. Immunity 2006; 24: 801-812.

40. O'Neill LA. Transflammation: when innate immunity meets induced pluripotency. Cell 2012; 151: 471-473.

41. Lee J, Sayed N, Hunter A, Au KF, Wong WH, Mocarski ES et al. Activation of innate immunity is required for efficient nuclear reprogramming. Cell 2012; 151: 547-558.

42. Ffrench B, O'Leary JJ, Gallagher MF. Therapeutically targeting epigenetic regulation of cancer stem cells. In: Grey S (ed). Epigenetic Cancer Therapy. Elselvier: Cambridge, MA, USA, 2015, pp 639-664.

43. Castaigne S, Chomienne C, Daniel MT, Ballerini P, Berger R, Fenaux P. All-trans retinoic acid as a differentiation therapy for acute promyelocytic leukemia. I. Cinical results. Blood 1990; 76: 1704-1709.

44. Chomienne C, Ballerune P, Bailtrand N, Daniel MT, Fenaux P, Castaigne S et al. All-trans retinoic acid in acute prolyelocytic leukemias. II. In vitro studies: structure-function relationship. Blood 1990; 76: 1710-1717.

45. Connolly R, Nguyen NK, Sukumar S. Molecular pathways: current role and future directions of the retinoic acid pathway in cancer prevention and treatment. Clin Cancer Res 2013; 19: 1651-1659.

46. Chefetz I, Alvero AB, Holmberg JC, Lebowitz N, Craveiro V, Yang-Hartwich Y et al. TLR2 enhances ovarian cancer stem cell self-renewal and promotes tumour repair and recurrence. Cell Cycle 2013; 12: 511-521.

47. Vencken SF, Sethupathy P, Blackshields G, Spillane C, Elbaruni S, Sheils $O$ et al. An integrated analysis of the Sox2 microRNA response program in human pluripotent and nullipotent stem cells. BMC Genomics 2014; 15: 711.

48. Gallagher MF, Flavin RJ, Elbaruni SA, Mclnerney JK, Smyth PC, Salley YM et al. Regulation of microRNA biosynthesis and expression in 2102Ep embryonal carcinoma stem cells is mirrored in ovarian serous adenocarcinoma patients. J Ovarian Res 2009; $2: 19$.

\section{Supplementary Information accompanies this paper on Cell Death and Differentiation website (http://www.nature.com/cdd)}

